\title{
Using weighted regression model for estimating cohort effect in age- period contingency table data
}

\author{
I-Shiang Tzeng ${ }^{1,2,3}$, Chau Yee Ng${ }^{4,5}$, Jau-Yuan Chen ${ }^{6,7}$, Li-Shya Chen ${ }^{8}$ and Chin- \\ Chieh Wu $\mathbf{u}^{9}$ \\ ${ }^{1}$ Department of Research, Taipei Tzu Chi Hospital, Buddhist Tzu Chi Medical Foundation, New Taipei City, Taiwan \\ ${ }^{2}$ Department of Statistics, National Taipei University, Taipei, Taiwan \\ ${ }^{3}$ Bachelor's Program of Financial Engineering and Actuarial Science, Feng Chia University, Taichung City, Taiwan \\ ${ }^{4}$ Department of Dermatology, Drug Hypersensitivity Clinical and Research Center, Chang Gung Memorial Hospital, Taipei, \\ Taiwan \\ ${ }^{5}$ School of Medicine, College of Medicine, Chang Gung University, Taoyuan, Taiwan \\ ${ }^{6}$ Department of Family Medicine, Chang-Gung Memorial Hospital, Linkou Branch, Taoyuan City, Taiwan \\ ${ }^{7}$ College of Medicine, Chang Gung University, Taoyuan, Taiwan \\ ${ }^{8}$ Department of Statistics, National Chengchi University, Taipei, Taiwan \\ ${ }^{9}$ Department of Emergency Medicine, Chang Gung Memorial Hospital, Keelung, Taiwan \\ Correspondence to: I-Shiang Tzeng, email: istzeng@gmail.com
}

Keywords: age-period-cohort; multiphase method; prediction; hepatocellular carcinoma

Received: May 18, $2017 \quad$ Accepted: February 22, $2018 \quad$ Published: April 13, 2018

Copyright: Tzeng et al. This is an open-access article distributed under the terms of the Creative Commons Attribution License 3.0 (CC BY 3.0), which permits unrestricted use, distribution, and reproduction in any medium, provided the original author and source are credited.

\section{ABSTRACT}

Background: Recently, the multiphase method was proposed to estimate cohort effects after removing the effects of age and period in age-period contingency table data. Hepatocellular carcinoma (HCC) is the most common primary malignancy of the liver and is strongly associated with cirrhosis, due to both alcohol and viral etiologies. In epidemiology, age-period-cohort (APC) model can be used to describe (or predict) the secular trend in HCC mortality.

Results: The confidence interval (CI) of the weighted estimates was found to be relatively narrow (compared to unweighted estimates). Moreover, for males, the mortality trend reverses itself during 2006-2010 was found from an increasing trend into a slightly deceasing trend. For females, the increasing trend reverses (earlier than males) itself during 2001-2005.

Conclusions: The weighted estimation of the regression model is recommended for the multiphase method in estimating the cohort effects in age-period contingency table data.

Impact: The regression model can be modified through the weighted average estimate of the effects with narrower CI of each cohort.

Methods: After isolating the residuals during the median polish phase, the final phase is to estimate the magnitude of the cohort effects using the regression model of these residuals on the cohort category with the weight equal to the occupied proportion according to the number of death of HCC in each cohort. 


\section{INTRODUCTION}

Evaluating disease and mortality patterns over time has become popular in understanding the utility of disease etiology in public health. However, the trend assessment of age-specific mortality presented inconsistent patterns between age groups. Birth cohort analyses are valuable in predicting future increases (or decreases) of diseases under the same pattern among birth cohorts. In epidemiology, one popular interpretation on the relationship between age, period, and cohort (APC) variables is that age and period interact to create unique generational experiences. Age effects are correlated with the outcome at various ages, such as deaths caused by cancer. Simultaneously, period effects influenced all ages over time. Birth cohort effects presented changes across groups with the same birth year who had the same outcome during the same period. Disease mortality is not only influenced by birth cohort effects but also affected by age and period. For example, if a person born in 1980 (i.e., birth cohort effects) is highly at risk of dying due to cardiovascular disease during his/her lifetime, it will take at least 30 years (i.e., period effects) for him/her to die during adulthood (i.e., age effects) at the beginning of 2010. Therefore, the conceptualization of cohort effects was proposed based on the interaction between age and period [1]. Although this conceptualization still has an exact linear relationship $($ age + cohort $=$ period $)$, exposures (predictors) are not intrinsic to birth cohorts. We would rather explain a cohort effect that existed while different disease distributions arise. However, as age + cohort $=$ period, these three variables are linear dependent, and unless additional constraints are imposed, APC model that estimates the linear effects of age, period, and cohort is non-identifiable. We have explained this problem and the potential constraints imposed in our previous publications [2-5]. However, methodological complexity is a barrier for many researchers. As previously mentioned, a cohort effect is conceptualized as a period effect that is differentially experienced through age-specific exposure to an event or cause (i.e., interaction) [6]. Addressing the identifiable problem in this conceptualization is unnecessary because cohort effects are not conceptualized independently from age and period. The median polish analysis has been used to estimate cohort effects under this conceptualization $[6,7]$.

Recently, the multiphase method was proposed by Keys and Li [6] and provides three phases of estimating cohort effects with minimal assumptions on the contingency table data. Moreover, the median polish does not rely on a specific distribution or structure and thus can be widely applied to various types of data, such as rates, log rates, proportions, and counts. The first phase is graphical representation. Graphs were conducted by age across periods or birth cohorts and even birth cohort across ages or periods. For example, we conduct a graph of rates of age across periods. If age-specific rates of different age groups varied mutually among different periods, then the period effect may exist in contingency table data. Cohort effect can also be present while age-specific rates of different age groups interacted mutually among different periods. The second phase involves median polish analysis to remove the additive effect of age and period by iteratively subtracting the median from each row and column. The final phase is regression procedure, which contain cohort effects and random error. We regressed these residuals on the cohort category (defined as an indicator variable) in a linear regression model with the aggregated count data in the format of contingency tables.

The median polish was developed to describe data in a two-way contingency table [8] and remove the additive influence of age (i.e., row) and period (i.e., column) by iteratively subtracting the median from each row and column. Selvin first applied the median polish to APC analysis [7]. This technique requires no assumptions about the distribution or structure of the data in a twoway contingency table. Consequently, it can be widely used for any type of data contained in a table without any assumption, such as suicide data [9]. APC model was also used to describe the secular trend in disease incidence or mortality [3]. The APC model usually assumed that age, period, and cohort have additive effects on the log transformation of disease/mortality rate.

Hepatocellular carcinoma (HCC) is the most common primary malignancy of the liver and is strongly associated with cirrhosis, due to both alcohol and viral etiologies [10]. Of all malignant tumors worldwide, HCC ranked fifth in terms of mortality in men (and the eighth in women). In Taiwan, it has been ranked as the first among all major cancers in men (and second in women) [11].

In this study, we investigated the longitudinal trends of HCC mortality data from the Vital Statistics as our demonstration. We evaluated the HCC mortality to identify the effects of age, period, and cohort and examined whether these effects varied by gender. This study aimed to use weighted average method to modify the multiphase method, in order to estimate the cohort effect. We also illustrated how to estimate the cohort effect using the multiphase method and compared the results to those estimated by proposed weighted average method.

\section{RESULTS}

Figures 1 and 2 show the HCC mortality rates among age and period groups. These fluctuations were more significant among men than women. The distribution of rates according to age shows that HCC mortality rates begin at 40-44 age group (see Figure 1). Note that HCC mortality rates rose gradually among those in $\geq 60$ age group (see Figure 2). However, HCC mortality rates based on age have considerably changed over time, which means that a significant cohort effect was hidden in the usual ageperiod cross-classified Vital Statistics table and will not apparent until the distant future. We perform the median 
polish procedure on the log-transformed HCC mortality rates. Tables 1 and 2 present the estimated cohort effects of the APC model on HCC mortality rates. Moreover, Table 3 also presents the age and period effects for both gender. Subsequently, Tables 1 and 2 report the weighted estimates obtained after calculating the weighted average procedure for both gender. According to the smallest deviance (compared to unweighted estimates) of confidence interval (CI) of the weighted estimates, the weighted estimates are better to fit the data. For men, in the left panel of Table 1 presents the cohort effects of the birth cohorts. The cohort effect increases from 0.75 (the earliest cohort effect in
1891 ) to 1.13 (the greatest cohort effect in 1936). For women, the cohort effect increases from 0.69 (the earliest cohort effect in 1891) to 1.17 (the greatest cohort effect in 1926). Note that the cohort effect significantly increased by approximately $51 \%$ and $68 \%$ compared to the cohort in 1891 for men and women, respectively. In the right panel of Table 1, the increase was evenly distributed. Here, the cohort effect increased from 0.71 (the earliest cohort effect in 1891) to 1.05 (the greatest cohort effect in 1936). For women, the increased distribution is presented similarly in the right panel of Table 2. The cohort effect increased from 0.65 (the earliest cohort effect in 1891) to 1.04 (the

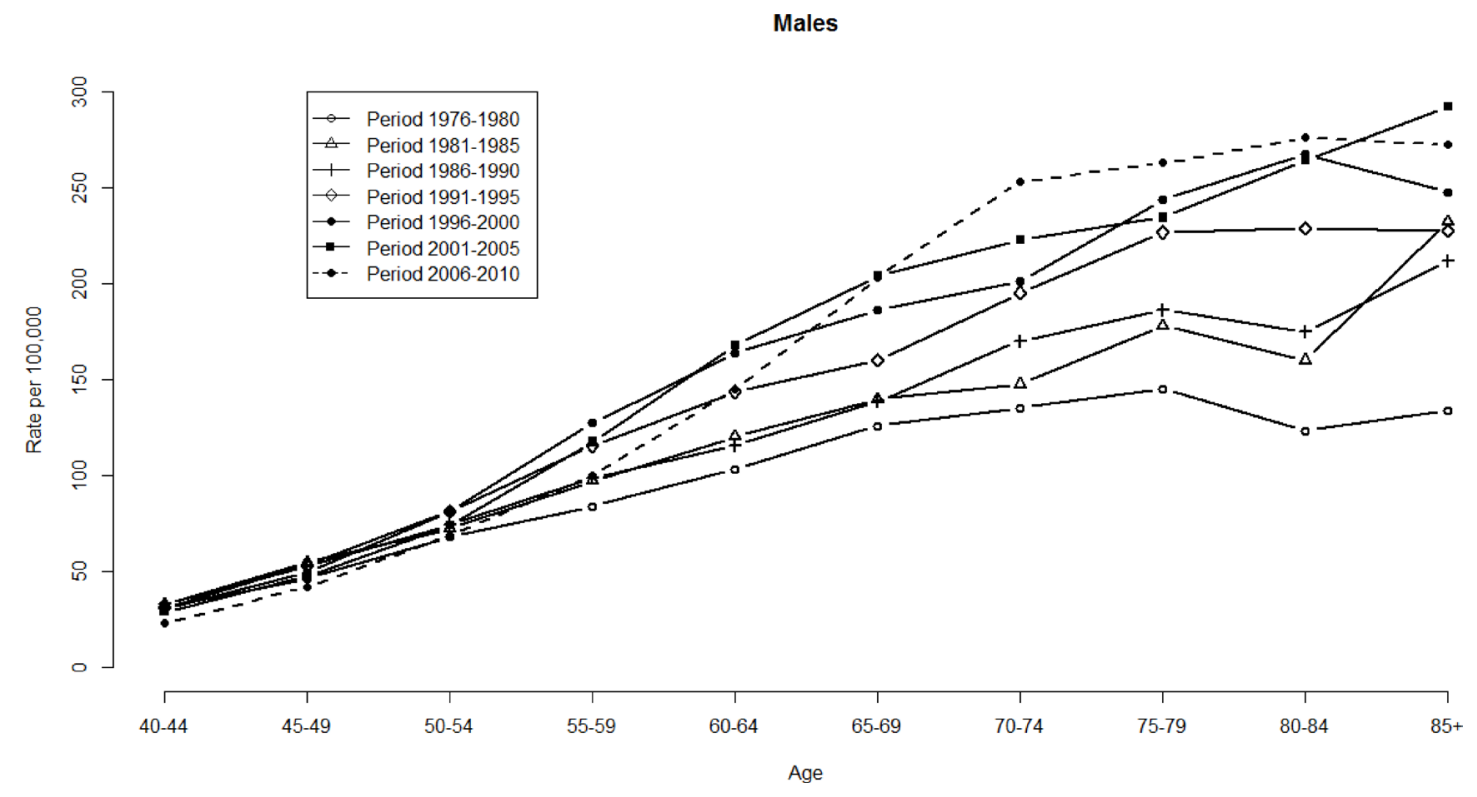

Figure 1: HCC mortality rates per 100,000 by age and period, males, Taiwan, 1976-2010.

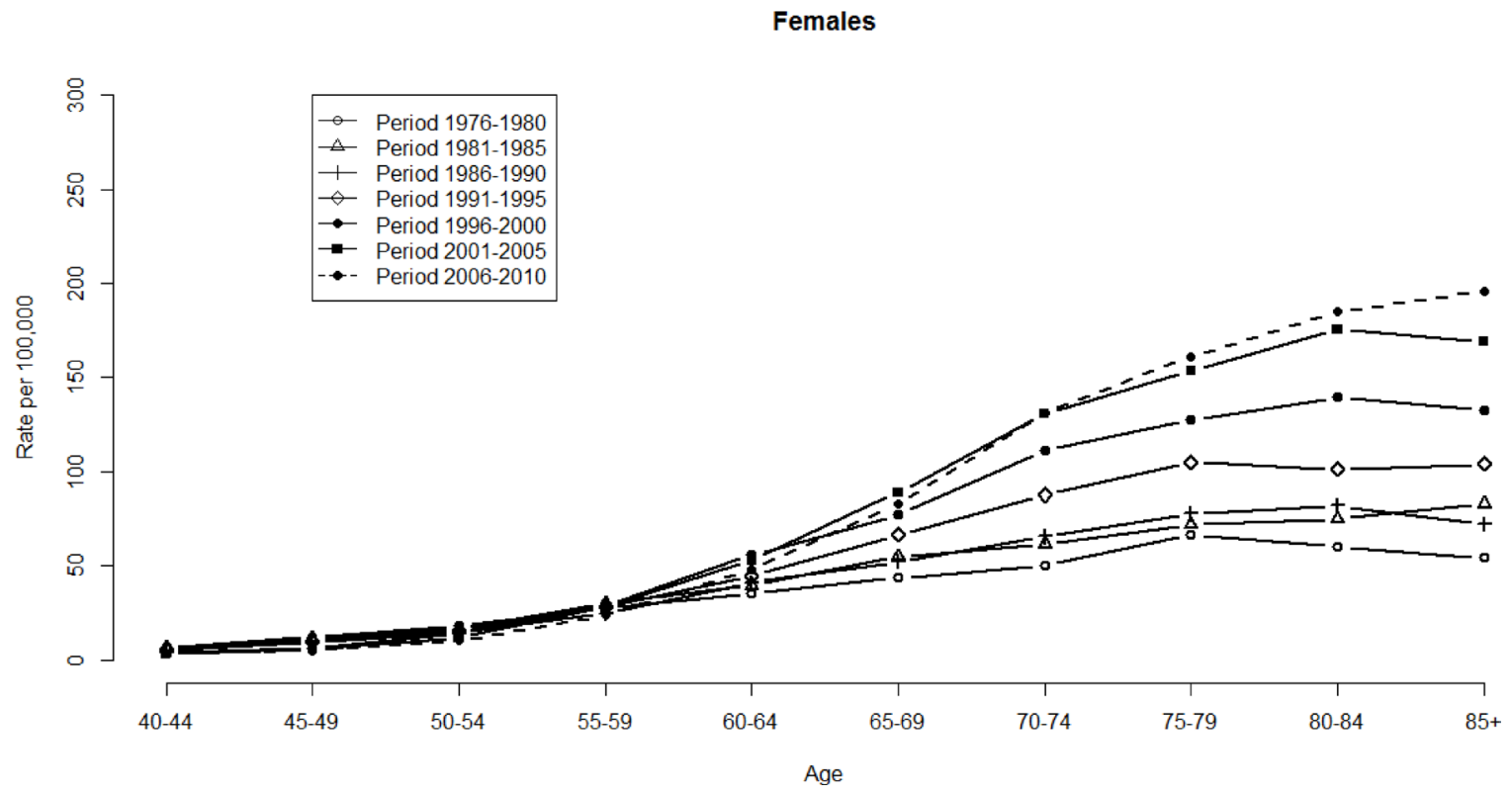

Figure 2: HCC mortality rates per 100,000 by age and period, females, Taiwan, 1976-2010. 
Table 1: Estimated rate ratios and $95 \%$ conference intervals for effect of birth cohort on hepatocellular carcinoma mortality of males in Taiwan, 1891-1966

\begin{tabular}{|c|c|c|c|c|}
\hline & \multicolumn{2}{|c|}{ Unweighted } & \multicolumn{2}{|c|}{ Weighted } \\
\hline & Effects & 95\% CI for Effects & Effects & 95\% CI for Effects \\
\hline \multicolumn{5}{|c|}{ Cohort } \\
\hline \multicolumn{5}{|c|}{$(1891 \sim 1966)$} \\
\hline 1891 & 0.75 & $0.62-0.89$ & 0.71 & $0.59-0.84$ \\
\hline 1896 & 0.90 & $0.82-0.99$ & 0.86 & $0.79-0.94$ \\
\hline 1901 & 0.91 & $0.85-0.97$ & 0.81 & $0.73-0.90$ \\
\hline 1906 & 0.93 & $0.88-0.98$ & 0.85 & $0.79-0.92$ \\
\hline 1911 & 0.97 & $0.92-1.02$ & 0.89 & $0.84-0.94$ \\
\hline 1916 & 1.03 & $0.98-1.08$ & 0.98 & $0.95-1.01$ \\
\hline 1921 & 1.01 & $0.97-1.06$ & 0.98 & $0.96-1.00$ \\
\hline 1926 & 1.01 & $0.97-1.05$ & 0.99 & $0.97-1.01$ \\
\hline 1931 & 1.06 & $1.01-1.10$ & 1.03 & $1.01-1.05$ \\
\hline 1936 & 1.13 & $1.09-1.18$ & 1.05 & $1.04-1.07$ \\
\hline 1941 & 1.09 & $1.04-1.14$ & 1.04 & $1.03-1.06$ \\
\hline 1946 & 1.00 & REF & 1.00 & REF \\
\hline 1951 & 0.87 & $0.82-0.92$ & 0.93 & $0.91-0.95$ \\
\hline 1956 & 0.82 & $0.77-0.88$ & 0.87 & $0.85-0.90$ \\
\hline 1961 & 0.77 & $0.70-0.85$ & 0.74 & $0.70-0.78$ \\
\hline 1966 & 0.68 & $0.57-0.82$ & 0.79 & $0.76-0.82$ \\
\hline
\end{tabular}

Note: $\mathrm{REF}=$ reference; $\mathrm{CI}=$ confidence interval.

Table 2: Estimated rate ratios and $95 \%$ conference intervals for effect of birth cohort on hepatocellular carcinoma mortality of females in Taiwan, 1891-1966

\begin{tabular}{|c|c|c|c|c|}
\hline & \multicolumn{2}{|c|}{ Unweighted } & \multicolumn{2}{|c|}{ Weighted } \\
\hline & Effects & 95\% CI for Effects & Effects & $95 \%$ CI for Effects \\
\hline \multicolumn{5}{|c|}{ Cohort } \\
\hline \multicolumn{5}{|c|}{ (1891 1966) } \\
\hline 1891 & 0.69 & $0.44-1.10$ & 0.65 & $0.41-1.03$ \\
\hline 1896 & 0.84 & $0.66-1.07$ & 0.78 & $0.61-1.00$ \\
\hline 1901 & 0.82 & $0.68-0.97$ & 0.73 & $0.56-0.95$ \\
\hline 1906 & 0.85 & $0.74-0.97$ & 0.78 & $0.68-0.89$ \\
\hline 1911 & 0.89 & $0.79-1.00$ & 0.86 & $0.79-0.93$ \\
\hline 1916 & 1.01 & $0.90-1.12$ & 0.98 & $0.94-1.02$ \\
\hline 1921 & 1.11 & $1.01-1.23$ & 1.04 & $1.01-1.07$ \\
\hline 1926 & 1.17 & $1.06-1.30$ & 1.03 & $1.01-1.05$ \\
\hline 1931 & 1.13 & $1.02-1.25$ & 1.02 & $1.00-1.03$ \\
\hline 1936 & 1.16 & $1.05-1.28$ & 1.02 & $1.00-1.03$ \\
\hline 1941 & 1 & REF & 1 & REF \\
\hline 1946 & 0.86 & $0.77-0.97$ & 0.97 & $0.95-0.99$ \\
\hline
\end{tabular}




\begin{tabular}{lllll}
1951 & 0.67 & $0.58-0.77$ & 0.86 & $0.84-0.89$ \\
1956 & 0.74 & $0.62-0.88$ & 0.79 & $0.75-0.83$ \\
1961 & 0.42 & $0.33-0.54$ & 0.52 & $0.47-0.57$ \\
1966 & 0.49 & $0.31-0.78$ & 0.49 & $0.44-0.54$ \\
\hline
\end{tabular}

Note: $\mathrm{REF}=$ reference; $\mathrm{CI}=$ confidence interval.

Table 3: Estimated age and period effects of among males and females, Taiwan, 1976-2010

\begin{tabular}{|c|c|c|}
\hline & Males & Females \\
\hline & Effect & Effect \\
\hline \multicolumn{3}{|l|}{ Period } \\
\hline 1976-1980 & -0.25 & -0.32 \\
\hline 1981-1985 & -0.10 & -0.15 \\
\hline 1986-1990 & -0.08 & -0.16 \\
\hline 1991-1995 & 0.04 & 0.02 \\
\hline 1996-2000 & 0.11 & 0.20 \\
\hline 2001-2005 & 0.16 & 0.25 \\
\hline 2006-2010 & 0.13 & 0.16 \\
\hline \multicolumn{3}{|l|}{ Age } \\
\hline $40-44$ & -1.37 & -2.01 \\
\hline $45-49$ & -0.83 & -1.36 \\
\hline $50-54$ & -0.41 & -0.90 \\
\hline $55-59$ & -0.09 & -0.29 \\
\hline $60-64$ & 0.12 & 0.20 \\
\hline $65-69$ & 0.32 & 0.53 \\
\hline 70-74 & 0.45 & 0.83 \\
\hline 75-79 & 0.54 & 1.02 \\
\hline 80-84 & 0.62 & 0.98 \\
\hline $85+$ & 0.67 & 1.00 \\
\hline Constant & -6.74 & -7.89 \\
\hline
\end{tabular}

greatest cohort effect in 1921). Thus, we observed that the mortality rate increasing by approximately $48 \%$ and $60 \%$ will become the peak value for men and women, respectively.

Among the birth cohorts, men born in 1936 exhibited the highest risk of HCC mortality (Table 1). Consequently, for weighted estimates, the effect was 1.05 (95\% CI: 1.04-1.07) for the 1936 birth cohort compared to the reference birth cohort in 1946 . However, a dramatically decreasing trend was observed for the earlier cohorts. Additionally, the effects were reversed after the 1936 cohort. Moreover, we plot the unweighted and weighted cohort effects with $95 \% \mathrm{CI}$ of men and women (Figures 3 and 4), respectively. Both figures show that almost all of the widths of $95 \%$ CI of weighted are shorter than that of unweighted cohort effects.
In this study, we limited our APC analysis of the median polish procedure to estimating cohort effects and $95 \%$ CIs of the HCC mortality. Based on this analysis, it appears that the residual errors $\left(\varepsilon_{i j k}\right)$ were close to zero.

\section{DISCUSSION}

Considering the time trend of HCC mortality, the conventional analysis using a simple linear extrapolation of the observed log age-adjusted rates may underestimate some important characteristics hidden in the data (such as the cohort effects) and facilitate prediction that are grossly missing. If we directly observe the long-term trends of HCC mortalities from 1976 to 2010 in Taiwan (Figure 5), no one with any reason will doubt that the trends, having been increasing for 35 years, will increase 
for the next few years. However, in fact the recent trend on HCC mortalities in Taiwan is decreasing and is driven by cohort effects (identified from the APC analysis), which as described decreased after the 1936 cohort. In this study, applying APC model allows advanced and more accurate warning for trend changes.

From a clinical viewpoint, hepatitis B virus (HBV) infection is an important health issue worldwide with high morbidity, approximately 2 billion people infected and 350 million suffering from chronic HBV infection [12]. The HBV infection can induce a wide range of clinical problems, from inactive carrier status to fulminate hepatitis, cirrhosis, or hepatocellular carcinoma. Injecting

Unweighted Cohort Effects

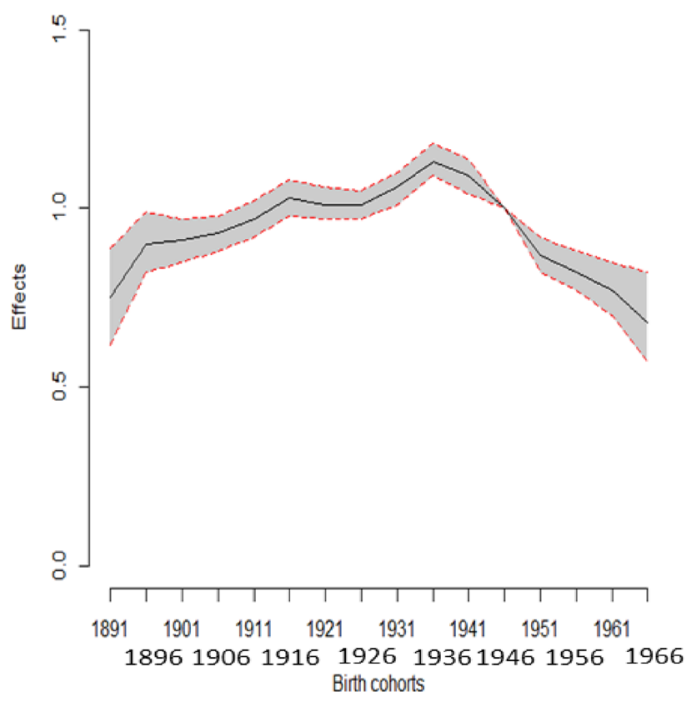

hepatitis $\mathrm{B}$ vaccine is the most effective prevention method. Based on the policy implications, the first worldwide hepatitis B mass vaccination program was implemented in 1984 in Taiwan [13]. They screened pregnant women for $\mathrm{HBsAg}$ and then $\mathrm{HBeAg}$. At first, the immunization program covered only infants of $\mathrm{HBsAg}$ carrier mothers in initially 2 years. From the third year of vaccination program, all infants were covered. Recently, the coverage rate of hepatitis B vaccine reached $99 \%$. After three vaccines, approximately $90-95 \%$ of the people will have life-long immunity. Note that the decline in pediatric HCC in Taiwan can be attributed to the contribution of this worldwide vaccination program. The APC estimation

Weighted Cohort Effects

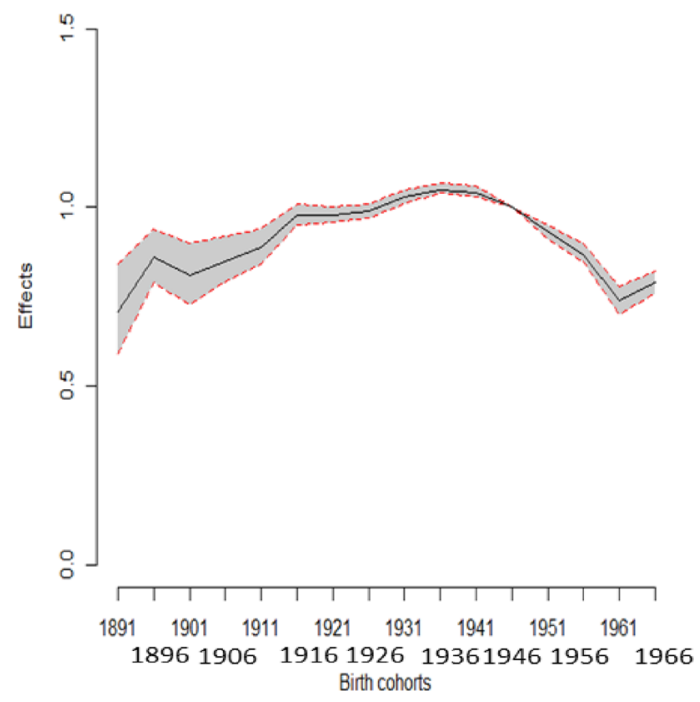

Figure 3: Plot of the unweighted and weighted effects with $95 \%$ confidence interval of males.
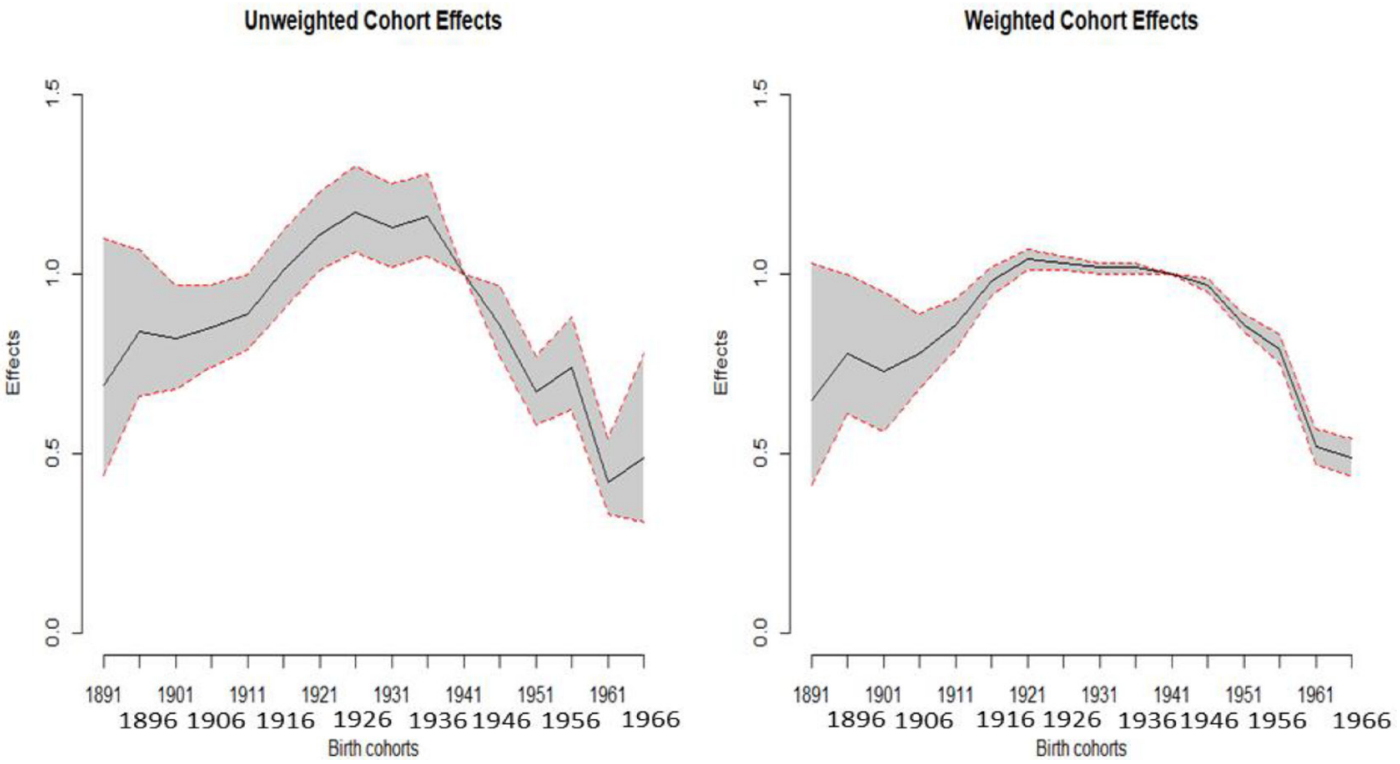

Figure 4: Plot of the unweighted and weighted effects with $95 \%$ confidence interval of females. 
described in this paper can have an advanced warning for these (increased) trend changes (to be decreased recently).

This study investigated trend of cohort effect through applying median polish procedure. The weighted estimates for modification of the regression model of these residuals then allow a weighted average estimate on the effect with narrower CI of each cohort. The results are reported in the form of cohort effects using the 1946 cohort, because these categories present the fewest changes in $\mathrm{HCC}$ mortality rates with cohort influence removed.

In most modeling methods (such as linear or nonlinear regression models), one of the common assumptions is that each real value of data provides equal information to estimate the parameters in a model which was undertaken. It means that the standard deviation of the error term is the constant underlying predictor variables. Based on our literature reviews, the assumption does not hold in modeling to empirically estimate the parameters. When we use weighted regression, the unknown parameters are estimated, a less weight is given for the less precise data points and more weights are given for the precise data points. The advantage is that weighted procedure can reduce the standard deviation of the estimator. However, the drawback of the weighted regression method is almost unknown in empirical practice. Because the exact weight is almost unknown, the estimated weight can be used to estimate the parameters. Moreover, experience shows that the weighting due to estimation does not change much and often does not affect regression analysis or its interpretation [14]. Theoretically, any disease with rates governed by age, period, and cohort effects is amenable for an APC model. Moreover, the weighted average estimates can be used for prediction [15-17]. If the CI is relatively narrow, the uncertainty is smaller, because the CI describes the uncertainty inherent in this estimate and range of values within which we can be reasonably sure that the true effect actually happens.

Several potential limitations of our study should be noted. First, we can only infer about the etiologies of the changes observed. The HCC mortality based on age, period, and cohort effects are re-amenable for an APC model. However, the presence of set assumptions for the median polish that we used should be noted in the present study. Second, APC analysis can be used extensively in the epidemiology field in populations of developing or recently developed countries, where long-running cohort studies are limited. Third, we do not have information from the aggregated format datasets to adjust confounders, such as comorbidities or lifestyle, in the APC model. Further studies using individual data is needed to solve this limitation. Fourth, we use the number of deaths due to $\mathrm{HCC}$ as the weight to modify the regression procedure in the multiphase method. Because the exact weight is almost unknown, the use of various weights may cause minor inflation among estimated cohort effects. Lastly, circumstances in which various APC estimation methods to address the non-identifiable problem may occur (e.g., Holford adopts the linear and curvature trends to tackle the non-identifiable problem [18]). Meanwhile, the median polish provides conceptual shift form complex assumption among APC model to estimate the cohort effect with a minimum of assumptions and easily applies a general format for contingency table.

In conclusion, the weighted estimation to modify the regression model then allows a weighted average effect with narrower CI of each cohort. In summary, the weighted estimation of the regression model is recommended for multiphase method to estimate the cohort effects in ageperiod contingency table data.
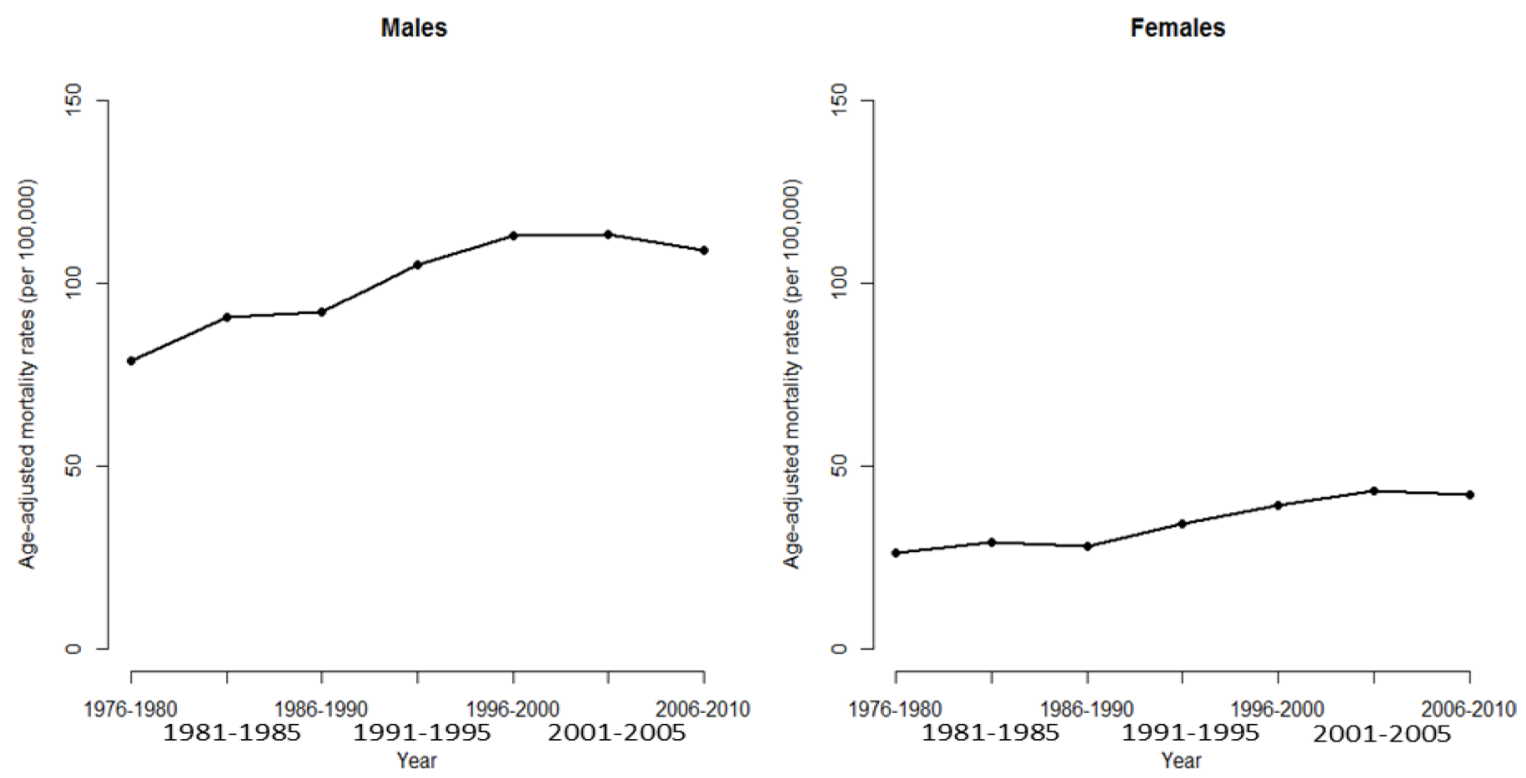

Figure 5: Age-adjusted mortality rate of death from hepatocellular carcinoma for men and women in Taiwan 
Table 4: Age-period contingency table of HCC mortality rate per 100,000 among males and females, Taiwan, $1976-2010$

\begin{tabular}{|c|c|c|c|c|c|c|c|}
\hline Males & 1976-1980 & 1981-1985 & 1986-1990 & 1991-1995 & 1996-2000 & $2001-2005$ & 2006-2010 \\
\hline $40-44$ & 31.41 & 33.10 & 33.20 & 31.24 & 30.40 & 29.10 & 23.26 \\
\hline $45-49$ & 46.50 & 55.13 & 52.88 & 53.61 & 49.79 & 47.66 & 42.25 \\
\hline $50-54$ & 68.11 & 72.47 & 74.56 & 81.73 & 81.65 & 74.19 & 69.12 \\
\hline $55-59$ & 84.12 & 97.32 & 98.96 & 115.57 & 127.55 & 117.70 & 100.19 \\
\hline $60-64$ & 103.58 & 120.45 & 115.71 & 143.74 & 164.14 & 168.20 & 145.35 \\
\hline $65-69$ & 126.15 & 140.12 & 138.76 & 160.29 & 186.58 & 204.31 & 203.50 \\
\hline $70-74$ & 135.44 & 147.79 & 170.44 & 195.56 & 201.40 & 223.00 & 253.46 \\
\hline $75-79$ & 145.41 & 178.22 & 186.70 & 226.86 & 243.87 & 234.63 & 263.24 \\
\hline $80-84$ & 123.63 & 160.33 & 175.09 & 229.23 & 267.55 & 264.61 & 276.39 \\
\hline $85+$ & 133.97 & 232.56 & 212.33 & 227.57 & 248.07 & 292.51 & 272.51 \\
\hline \multicolumn{8}{|c|}{ Females } \\
\hline $40-44$ & 6.75 & 6.14 & 4.90 & 5.11 & 3.46 & 2.88 & 3.03 \\
\hline $45-49$ & 11.99 & 11.10 & 9.21 & 9.76 & 6.49 & 5.76 & 4.90 \\
\hline $50-54$ & 18.20 & 16.23 & 14.78 & 15.53 & 14.15 & 11.78 & 10.20 \\
\hline $55-59$ & 27.57 & 30.06 & 24.45 & 28.43 & 28.65 & 28.04 & 23.68 \\
\hline $60-64$ & 35.23 & 39.48 & 41.09 & 44.75 & 55.75 & 52.97 & 48.01 \\
\hline $65-69$ & 43.49 & 54.68 & 52.09 & 66.36 & 76.92 & 89.05 & 82.80 \\
\hline $70-74$ & 50.40 & 61.49 & 65.48 & 87.47 & 111.46 & 130.60 & 131.32 \\
\hline $75-79$ & 66.47 & 72.02 & 77.57 & 105.09 & 127.37 & 153.26 & 160.98 \\
\hline $80-84$ & 60.12 & 74.79 & 81.83 & 101.26 & 139.41 & 175.41 & 184.90 \\
\hline $85+$ & 54.22 & 82.91 & 71.93 & 103.98 & 132.42 & 169.12 & 196.03 \\
\hline
\end{tabular}

\section{MATERIALS AND METHODS}

\section{Data source}

To illustrate the calculations, we used the HCC mortality data from 1976 to 2010 for men and women in Taiwan, which were obtained from individual health records of the Ministry of Health and Welfare (MOHW). HCC mortality was classified based on the International Classification of Disease (ICD) Code 150. The mortality data were available for 10 five-year age groups (40-44, 45-49, 50-54, 55-59, 60-64, 65-69, 70-74, 75-79, 8084, and 85+), 7 five-year time periods (1976-1980, 19811985, 1986-1990, 1991-1995, 1996-2000, 2001-2005, and 2006-2010), and 16 birth cohorts (mid-cohort years: 1891, 1896, 1901, 1906, 1911, 1916, 1921, 1926, 1931, 1936,1941, 1946, 1951, 1956, 1961, and 1966). In Table 4 , we present an age-period contingency table format for the HCC mortality of men and women. From these, we calculated the age-specific and the age-adjusted (using the 2000 World Standard Population) mortality rates [19].

Let the mortality rate of the $i^{\text {th }}$ age group and the $j^{\text {th }}$ period group be denoted by $\lambda_{i j}$ The APC model is as follows:

$$
\begin{aligned}
\log \lambda_{i j}=\mu+\alpha_{i}+\beta_{j}+\gamma_{k}, \quad i & =1,2, \ldots, I, \\
j & =1,2, \ldots, J,(\mathrm{Eq}-1) \\
k & =j-i+I,
\end{aligned}
$$

where the intercept term is represented by $\mu$, the age effects by $\alpha_{i}$, the period effects by $\beta_{j}$, and the cohort effects by $\gamma_{K}$. The following constraints are used:

$$
\sum_{i} \alpha_{i}=\sum_{j} \beta_{j}=\sum_{k} \gamma_{k}=0
$$

\section{The multiphase method in estimating cohort effects}

The multiphase method includes three-phase processes that concretized the estimation of the cohort effect as a partial interaction in age-period contingency table data $[6,9]$. The natural $\log$ rate $\left(\lambda_{i j}\right)$ is established using the log-additive effect as a constant term plus age, period effect, and multiplicative interaction term, which 
can be regarded as a fully saturated model that includes systematic and unsystematic components (random error). The systematic component contains cohort effect. After isolating the residuals from the median polish phase, the final phase is to estimate the magnitude of cohort effects through regression of these residuals $\left(\varepsilon_{k}\right)$ on the cohort category (cohort is an indicator variable entered as a collection of the $\mathrm{m}+\mathrm{n}-1$ cohorts as $\mathrm{k}=1,2, \ldots, \mathrm{m}+\mathrm{n}-1$ )

$$
\varepsilon_{k}=\gamma_{k}+\varepsilon_{i j k}
$$

The $\varepsilon_{k}$ is established using a vector of cohort effects $\left(\gamma_{k}\right)$ and error terms $\left(\varepsilon_{i j k}\right)$, where $\varepsilon_{i j k}$ represented the error terms unmeasured as $i$ age, $j$ period, and $k$ cohort categories.

According to the cohort-specific mortality by age that calculated as removed and unremoved cohort influence to decide reference categories. Reference categories of cohort had a minimum difference in cohortspecific mortality between with and without cohort influence. After subtracting residuals form contingency data, we can use the residual to calculate the log-additive rate (without cohort effect) with multiplying factor $e^{- \text {(residual) }}$ to the rate each age and period group. Then, take the ratio of log-additive rate without and with cohort effects for each cohort. If the rate ratio of the cohort is close to one, then it is determined as the referent birth cohort. The referent birth cohort can be determined based on the slight variation of its rate after removing the influencing factors.

\section{Weighted average}

For birth cohorts with members that achieved the cohort, let $W_{k}$ denote the weight of the $k^{\text {th }}$ cohort category:

$$
\varepsilon_{k}=W_{k} \times \gamma_{k}+\varepsilon_{i j k}
$$

However, the common assumption is that each cohort across $i$ age and $j$ period of data provides equal information (i.e., equally weighted) for the estimation of cohort effects in a model while the weighting factor is generally unknown. Empirically, the equally weighted assumption is usually violated while modeling to estimate the cohort effect. The empirical weighting factor most widely used is the number of death [20]. Each of these weights can be applied to the regression equation. The weighted average of the cohort effect can be performed via the weight equal to the occupied proportion according to the number of deaths due to HCC in each cohort.

To check model fitness and furthermore we plot deviance residuals which from the null model, the age model, the age-period model, and then to the APC model (under the proposed weighted method) progressively (Supplementary Figure 1).

\section{ACKNOWLEDGMENTS}

The authors thank Mr. Chang Chih Shih who employed in the Department of Statistics, Ministry of Health and Welfare, Taiwan, for helpful comments and suggestions on the Vital Statistics.

\section{CONFLICTS OF INTEREST}

We declared no conflicts of interest.

\section{REFERENCES}

1. Greenberg BG, Wright JJ, Sheps CG. A technique for analyzing some factors affecting the incidence of syphilis. Journal of the American Statistical Association. 1950; 45:373-99.

2. Liu SH, Tzeng IS, Hsieh TH, Huang YC. Associations between excessive adiposity and seroprevalence of herpes simplex virus type 1 and type 2 among US adults: a population-based age-period-dohort analysis. BMJ. 2016; 6:e012571.

3. Tzeng IS, Lee WC. Forecasting hepatocellular carcinoma mortality in Taiwan using an age-period-cohort model. Asia Pac J Pub Health. 2015; 27:NP65-73.

4. Tzeng IS, Liu SH, Chiou YT, Huang CH, Lee CJ, Chien CY, Hsu SC, Weng YM, Chen KF, Chen JC. Predicting emergency departments visit rates from septicemia in Taiwan using an age-period-cohort model, 1998 to 2012. Medicine. 2016; 95:e5598.

5. Tzeng IS, Yue JC, Lee YT. An empirical study on death rate estimation and exploration of annuity-related issues. Insurance Monograph. 2016; 32:105-31.

6. Keyes KM, Li G. A multiphase method for estimating cohort effects in age-period contingency table data. Ann Epidemiol. 2010; 20:779-85.

7. Selvin S. Statistical analysis of epidemiologic data: New York: Oxford University Press. 1996.

8. Tukey J. Exploratory data analysis. Reading: MS: AddisonWesley Publishing Company. 1977.

9. Légaré G, Hamel D. An age-period-cohort approach to analyzing trends in suicide in Quebec between 1950 and 2009. Can J Pub Health. 2013; 104:e118-23.

10. Pinter M, Trauner M, Peck-Radosavljevic M, Sieghart W. Cancer and liver cirrhosis: implications on prognosis and management. ESMO Open. 2016; 1:e00042.

11. McGlynn KA, Tsao L, Hsing AW, Devesa SS, Fraumeni JF Jr. International trends and patterns of primary liver cancer. Int J Cancer. 2001; 94:290-6.

12. Lavanchy D. Hepatitis B virus epidemiology, disease burden, treatment, and current and emerging prevention and control measures. J Viral Hepat. 2004; 11:97-107. 
13. Chang MH, Chen CJ, Lai MS, Hsu HM, Wu TC, Kong MS, Liang DC, Shau WY, Chen DS. Universal hepatitis B vaccination in Taiwan and the incidence of hepatocellular carcinoma in children. Taiwan Childhood Hepatoma Study Group. N Eng J Med. 1997; 336:1855-9.

14. da Silva CP, Emídio ES, de Marchi MR. Method validation using weighted linear regression models for quantification of UV filters in water samples. Talanta. 2015; 131:221-7.

15. Dawes RM. The robust beauty of improper linear models in decision making. Am Psychol. 1979; 34:571-82.

16. Dawes RM, Corrigan B. Linear models in decision making. Psychological Bulletin. 1974; 81:95-106.
17. Einhorn HJ, Hogarth RM. Unit weighting schemes for decision making. Organizational Behavior and Human Performance. 1975; 13:171-92.

18. Holford TR. The estimation of age, period and cohort effects for vital rates. Biometrics. 1983; 39:311-324.

19. Ahmad OB, Boschi-Pinto C, Lopez AD, Murray CJL, Lozano R, Inoue M. Age Standardization of Rates: A New WHO Standard. GPE Discussion Paper Series. 2005; 31.

20. Wilmoth JR. Computational Methods for Fitting and Extrapolating the Lee-Carter Model of Mortality Change. 1993; Technical Report, University of California, Berkeley. 